\title{
9 ATUAÇÃO DO ENFERMEIRO NO ATENDIMENTO AO PÚBLICO INFANTO- JUVENIL EM SOFRIMENTO MENTAL: UMA REVISÃO INTEGRATIVA
}

\author{
| Rachel Santos ${ }^{1}$; Isabela Takeshita ${ }^{2}$; Franciéle Cardoso ${ }^{3}$; Lilian Torres ${ }^{4} \mid$
}

\section{RESUMO}

CONTEXTO: A qualidade da assistência de enfermagem em saúde mental é essencial na vida das crianças e adolescentes. OBJETIVO: Descrever a atuação do enfermeiro no cuidado ao público infantojuvenil em sofrimento mental.

MÉTODO: Revisão integrativa da literatura nas bases de dados BVS e EBSCO, a partir de descritores selecionados. A amostra constituiu-se de 15 artigos, publicados entre 2013 a 2017, disponíveis na íntegra e nos idiomas português, inglês e espanhol. RESULTADOS: A atuação do enfermeiro perpassa pela gestão e organização do cuidado e considera o olhar individualizado e respeitoso. Busca-se a promoção da autonomia e ressocialização desses jovens e suas famílias, a partir da inclusão do familiar/ cuidador no planejamento do cuidado.

CONCLUSÃO: O olhar ampliado e a variedade de intervenções desempenhadas pelo enfermeiro torna sua prática complexa e fundamental para a qualidade de vida desses jovens. O diferencial em sua atuação está no conhecimento aberto, reflexivo e cientificamente embasado.

PALAVRAS-CHAVE: Psiquiatria infantil; Psiquiatria do adolescente; Serviços de saúde mental; Enfermagem

\section{RESUMEN}

“Actuación del enfermero en la atención al público infantojuvenil en sufrimiento mental: Una revisión integrativa"

CONTEXTO: La calidad de la asistencia de enfermería en salud mental es esencial en la vida de los niños y adolescentes.

OBJETIVO: Describir la actuación del enfermero en el cuidado al público infantojuvenil en sufrimiento mental.

MÉTODOLOGÍA: Revisión integrativa de la literatura en las bases de datos BVS y EBSCO, a partir de descriptores seleccionados. La muestra consistió en 15 artículos publicados entre 2013 a 2017, totalmente disponible electrónicamente en portugués, inglés y español.

RESULTADOS: La actuación del enfermero pasa por la gestión y organización del cuidado y considera la mirada individualizada y respetuosa. Se busca la promoción de la autonomía y resocialización de estos jóvenes y sus familias, a partir de la inclusión del familiar / cuidador en la planificación del cuidado.

CONCLUSIÓN: La mirada ampliada y la variedad de intervenciones desempeñadas por el enfermero hacen su práctica compleja y fundamental para la calidad de vida de esos jóvenes. El diferencial en su actuación está en el conocimiento abierto, reflexivo y científicamente embasado.

DESCRIPTORES: Psiquiatría infantil; Psiquiatría del adolescente; Servicios de salud mental; Enfermería

\begin{abstract}
"Nurses' performance in the care of children and adolescents in mental suffering: An integrative review"

BACKGROUND: The quality of nursing care in mental health is essential in the lives of children and adolescents.

AIM: To describe the nurse's role with the child and adolescent public in mental suffering.

METHOD: Integrative literature review on the VHL and EBSCO databases, from selected descriptors. The sample consisted of 15 articles, published between 2013 and 2017, available in full electronic form and in the Portuguese, English and Spanish languages. RESULTS: Nurses work through the management and organization of care and consider the individualized and respectful look. What is sought is the promotion of the autonomy and resocialization of these young people and their families, from the inclusion of the caregiver in the planning of care.

CONCLUSION: The expanded look and variety of interventions performed by nurses makes their practice complex and fundamental to the quality of life of these young people. The differential in its performance lies in open, reflective and scientifically grounded knowledge.
\end{abstract}

KEYWORDS: Child psychiatry; Adolescent psychiatry; Mental health services; Nursing

Submetido em 30-09-2019

Aceite em 12-12-2019

1 Enfermeira, Especialista em Auditoria em Enfermagem, Enfermeira de Referência na Fundação FIAT Saúde e Bem Estar, rachelsilvasantosmg@gmail.com

2 Enfermeira, Especialista em Enfermagem do Trabalho, Mestre em Enfermagem, Docente na Faculdade Ciências Médicas de Minas Gerais, isabelamie@gmail.com

3 Enfermeira, Especializanda em UTI Geral e Assistência Intensiva ao Paciente Crítico, francieleavilaboeira@gmail.com

4 Enfermeira, Mestre e Doutor em Ciências, lilian.torres0806@gmail.com

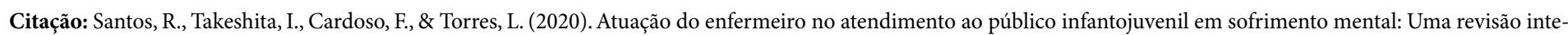
grativa. Revista Portuguesa de Enfermagem de Saúde Mental (24), 69-78. 


\section{INTRODUÇÃO}

A Reforma Sanitária, o Sistema Único de Saúde e o Estatuto da Criança e do Adolescente, em 1990, redefiniram as políticas públicas de assistência à saúde ao público infantojuvenil no Brasil (Reis et al., 2010). Em especial, a Reforma Psiquiátrica, com um novo modelo de atenção, advogou pela descentralização do cuidado e extinção dos hospitais psiquiátricos (Secretaria de Atenção à Saúde [SAS], 2005a). Os serviços comunitários, com estratégias de mobilização específica, procuraram incluir no seio familiar e na sociedade os indivíduos em sofrimento mental (Machado et al., 2014).

O sofrimento mental infantojuvenil, em metade dos casos, tem seu início antes dos 14 anos (Naughton, Maybery, \& Goodyear, 2018). Tal população representa um terço do total porém, mais que números, constitui o grupo mais vulnerável dos pontos de vista físico, econômico e social (Gogineni, Rothe, \& Pumariega, 2019).

Reconhecer e compreender a importância da saúde mental de crianças e adolescentes para a saúde pública tem recebido ênfase de vários organismos internacionais (Gogineni, Rothe, \& Pumariega, 2019) e favorecido, no Brasil, o desenvolvimento de diretrizes governamentais, com serviços articulados em rede e com inclusão social. Os exemplos são as Unidades Básicas de Saúde, a Estratégia de Saúde da Família (ESF), os Centros de Atenção Psicossocial Infantojuvenil (CAPSi), os ambulatórios e os hospitais gerais (Reis et al., 2010).

O CAPSi, em especial, destina-se a crianças e adolescentes e tem se revelado fundamental na assistência em saúde mental (SAS, 2005a \& 2005b). Trata-se de desconstruir o modelo hospitalocêntrico, promover um serviço que respeite as características regionais e que, efetivamente, insira os indivíduos em atividades educativas e culturais em seu próprio contexto (Machado et al., 2014; Thiengo, Cavalcante, \& Lovisi, 2014).

Um dos documentos governamentais, em 2002, define e estabelece diretrizes para os CAPSi, como atendimento individual (medicamentoso, psicoterápico e de orientação) e em grupos (psicoterapia, grupo operativo e atividades de suporte social), além das oficinas terapêuticas, visitas e atendimentos domiciliários. Incluem-se acolhimento familiar, atividades comunitárias, com enfoque na integração da criança e do adolescente e ações intersetoriais, nas áreas de assistência social, educação e justiça (Ministério da Saúde, 2002). Apesar de avanços nas estruturas burocrática e organizativa, sua finalidade, entretanto, ainda é desconhecida, sobretudo, pelo profissional de saúde (Cunha, Borges, \& Bezerra, 2017).
A integração dos centros de atenção com a ESF tornase indispensável, nessa perspectiva, para o cuidado integral às crianças e adolescentes em sofrimento mental. O caminho, árduo e desconhecido, conclama a união de todos os atores do sistema para encontra-lo e o enfermeiro tem papel relevante na integração desejada (Delfini, Reis, 2012).

Ademais, no CAPSi, esse profissional coordena a equipe, planeja e avalia a assistência de enfermagem, individual ou em grupo. Acresce-se o desenvolvimento e manutenção do ambiente terapêutico, o cuidado propriamente dito ao indivíduo/família/comunidade e a cooperação na formação e educação permanente dos profissionais. No cotidiano, o enfermeiro realiza pré-consulta e triagem, consulta de enfermagem, coordena grupos terapêuticos, reuniões em geral e orientações sobre uso de medicamentos (Secretaria Executiva, 2003).

Diante desse contexto de relevância dos serviços especializados ou mesmo da ESF, no atendimento a crianças e adolescentes em sofrimento mental, questiona-se: qual a atuação do enfermeiro junto ao público infantojuvenil no cuidado em saúde mental?

O estudo torna-se relevante ao se considerar que os CAPSi tem se expandido em âmbito nacional. Em 2006, havia 75 centros e, em 2014, mais de 200 unidades, segundo o Boletim Informativo Saúde Mental em Dados, de $n^{\circ} 12$, do Ministério da Saúde (SAS, 2015). É essencial que o enfermeiro conheça em profundidade as suas atribuições, a partir da sua formação histórica/cultural, em que o cuidado procura entender as possibilidades terapêuticas. A sua abordagem, como educador e facilitador do processo de aprendizagem e adaptação contribuiria para a qualificação do cuidado em saúde mental. O objetivo proposto é descrever a atuação do enfermeiro no atendimento ao público infantojuvenil em sofrimento mental.

\section{MÉTODOS}

Revisão integrativa da literatura. Tipo de pesquisa que representa um método específico para a compreensão mais abrangente de um dado fenômeno, a partir do que já está posto, enquanto conhecimento (Botelho, Cunha, Macedo, \& 2011). São percorridas seis etapas: elaboração do problema, colheita de informações, avaliação crítica, análise e interpretação dos dados recolhidos, além da apresentação pública (Mendes, Silveira, Galvão, \& 2008). A população do estudo foi composta por publicações nacionais e internacionais das bases de dados da Biblioteca Virtual de Saúde (BVS) e EBSCO Information Services. 
Os Descritores em Ciências da Saúde coincidiram com aqueles contidos no Medical Subject Headings: "Child psychiatry", "Adolescent psychiatry”, "Community mental health services", "Nursing”, “Child” e "Mental health". Foram incluídos os artigos publicados entre janeiro de 2013 a dezembro de 2017, além de publicações disponíveis na íntegra e nos idiomas inglês, português e espanhol. Após os cruzamentos dos descritores, identificou-se uma população de 148 artigos (Tabela 1).

Tabela 1 - Cruzamento de descritores para busca na literatura

\begin{tabular}{|l|c|c|c|}
\hline Descritores e cruzamentos & EBSCO & BVS & TOTAL \\
\hline $\begin{array}{l}\text { Psiquiatria Infantil "AND" Psiquia- } \\
\text { tria do Adolescente }\end{array}$ & 14 & 0 & 14 \\
\hline $\begin{array}{l}\text { Serviços de Saúde Mental "AND" } \\
\text { Enfermagem }\end{array}$ & 11 & 49 & 60 \\
\hline $\begin{array}{l}\text { Psiquiatria do Adolescente "AND" } \\
\text { Psiquiatria Infantil "AND" Enfer- } \\
\text { magem }\end{array}$ & 1 & 0 & 1 \\
\hline $\begin{array}{l}\text { Enfermagem "AND" Psiquiatria } \\
\text { Infantil }\end{array}$ & 5 & 0 & 5 \\
\hline $\begin{array}{l}\text { Enfermagem "AND" Psiquiatria } \\
\text { Infantil "AND" Saúde Mental }\end{array}$ & 0 & 50 & 50 \\
\hline $\begin{array}{l}\text { Serviços de Saúde Mental "AND" } \\
\text { Psiquiatria Infantil "AND" Enfer- } \\
\text { magem }\end{array}$ & 0 & 12 & 12 \\
\hline $\begin{array}{l}\text { Serviços de Saúde Mental "AND" } \\
\text { Psiquiatria Infantil }\end{array}$ & 0 & 6 & 6 \\
\hline TOTAL & 31 & 117 & 148 \\
\hline
\end{tabular}

Fonte: Dados da Pesquisa

A totalidade de 148 estudos foi analisada em conjunto por três dos pesquisadores em relação à pertinência de títulos e resumos, sendo selecionados 23 artigos, mediante a concordância de todos os avaliadores, nesta fase, anterior à leitura na íntegra de cada pesquisa. Em seguida, cada pesquisador leu integralmente a seleção. $\mathrm{Na}$ discussão conjunta, momento posterior em que os pesquisadores apresentaram sua síntese de leitura, cada artigo foi definido como integrante da amostra ou não, mediante a sua capacidade de resposta à questão norteadora. Quando a análise não se apresentou unânime, nova leitura e discussão se realizou, dessa vez em grupo, para a definição final. Tal processo eliminou oito estudos por não responderem à questão norteadora. A amostra constituiu-se, portanto, de 15 publicações, sete na EBSCO e, oito, na BVS (Tabela 2).

Tabela 2 - População e amostra dos bancos de dados utilizados

\begin{tabular}{|c|c|c|c|}
\hline População & $\begin{array}{c}\text { Leitura de títulos } \\
\text { e resumos }\end{array}$ & $\begin{array}{c}\text { Leitura na } \\
\text { íntegra }\end{array}$ & Amostra \\
\hline 148 & 23 & 23 & 15 \\
\hline
\end{tabular}

Fonte: Dados da Pesquisa
Após a leitura cuidadosa da amostra realizou-se, primeiramente, a avaliação da qualidade metodológica dos estudos selecionados, por meio das ferramentas publicadas, em 2014, no Manual do Instituto Joana Briggs (The Joanna Briggs Institute, 2014). Os instrumentos foram aplicados e cada publicação deveria atender a, pelo menos, $50 \%$ dos itens que compunham os critérios de avaliação das evidências apresentadas, para sua permanência na amostra.

Em seguida, realizou-se a análise de cada artigo, de forma individual, pelos autores para identificação de respostas à questão norteadora. Finalmente, todos os pesquisadores se reuniram para a organização conjunta dos resultados encontrados.

\section{RESULTADOS}

O exame dos 15 artigos selecionados considerou as variáveis relacionadas aos autores, às publicações e àquela, de interesse, ou seja, a questão de pesquisa. Agruparam-se as ideias comuns sobre a atuação do enfermeiro na saúde mental do público infantojuvenil.

\section{Caracterização dos autores da seleção de artigos}

No total, são 67 autores, sendo 47 (70\%), enfermeiros. Quanto à qualificação, 29 (43\%) são doutores, 18 (27\%) graduados, 12 (18\%) mestres e, oito (12\%), especialistas. Mais da metade $(53 \%, \mathrm{n}=8)$ se qualificou no Brasil e 42 (63\%) atuam como docentes (Quadro 1).

Quadro 1 - Caracterização dos autores da amostra

\begin{tabular}{|l|l|l|l|l|}
\hline Autor & Profissão & $\begin{array}{l}\text { Área de } \\
\text { Atuação }\end{array}$ & $\begin{array}{l}\text { País de } \\
\text { qualificação }\end{array}$ & Qualificação \\
\hline $\begin{array}{l}\text { Barkmann } \\
\text { et al ., 2015 }\end{array}$ & $\begin{array}{l}3 \text { Enfer- } \\
\text { meiros } \\
2 \text { Psicólogos } \\
1 \text { Médico }\end{array}$ & 6 Docentes & Alemanha & 6 Doutores \\
\hline $\begin{array}{l}\text { Beentjes, } \\
\text { Goos- } \\
\text { sens, \& } \\
\begin{array}{l}\text { Jongerden, } \\
2016\end{array}\end{array}$ & $\begin{array}{l}3 \text { Enfer- } \\
\text { meiros }\end{array}$ & 3 Docentes & Holanda & $\begin{array}{l}2 \text { Doutores } \\
1 \text { Especialista }\end{array}$ \\
\hline $\begin{array}{l}\text { Dutra, } \\
\text { Bossato, \& } \\
\text { Oliveira, } \\
2017\end{array}$ & $\begin{array}{l}3 \text { Enfer- } \\
\text { meiros }\end{array}$ & $\begin{array}{l}3 \text { Assisten- } \\
\text { ciais }\end{array}$ & Brasil & 3 Graduados \\
\hline $\begin{array}{l}\text { Gloff et al., } \\
2015\end{array}$ & $\begin{array}{l}3 \text { Enfer- } \\
\text { meiros } \\
1 \text { Médico }\end{array}$ & $\begin{array}{l}3 \text { Assisten- } \\
\text { ciais } \\
1 \text { Docente }\end{array}$ & $\begin{array}{l}\text { Estados } \\
\text { Unidos }\end{array}$ & $\begin{array}{l}2 \text { Doutores } \\
1 \text { Mestre } \\
1 \text { Especialista }\end{array}$ \\
\hline $\begin{array}{l}\text { Hirota et } \\
\text { al., 2015 }\end{array}$ & $\begin{array}{l}6 \text { Enfer- } \\
\text { meiros } \\
3 \text { Médicos } \\
1 \text { Psicólogo }\end{array}$ & $\begin{array}{l}10 \text { Docen- } \\
\text { tes }\end{array}$ & $\begin{array}{l}\text { Coreia do } \\
\text { Sul }\end{array}$ & $\begin{array}{l}2 \text { Doutores } \\
3 \text { Mestres } \\
2 \text { Especial- } \\
\text { istas } \\
3 \text { Graduados }\end{array}$ \\
\hline
\end{tabular}




\begin{tabular}{|c|c|c|c|c|}
\hline $\begin{array}{l}\text { Macedo } \\
\text { Monteiro } \\
\text { et al., 2015 }\end{array}$ & $\begin{array}{l}5 \text { Enfer- } \\
\text { meiros }\end{array}$ & $\begin{array}{l}2 \text { Docentes } \\
3 \text { Assisten- } \\
\text { ciais }\end{array}$ & Brasil & $\begin{array}{l}1 \text { Doutor } \\
1 \text { Mestre } \\
3 \text { Graduados }\end{array}$ \\
\hline $\begin{array}{l}\text { Ma- } \\
\text { chineski, } \\
\text { Schneider, } \\
\text { \& Camatta, } \\
2013\end{array}$ & $\begin{array}{l}3 \text { Enfer- } \\
\text { meiros }\end{array}$ & 3 Docentes & Brasil & 3 Doutores \\
\hline $\begin{array}{l}\text { Martinez, } \\
\text { Tocantins, } \\
\text { \& Souza, } \\
2013\end{array}$ & $\begin{array}{l}3 \text { Enfer- } \\
\text { meiros }\end{array}$ & $\begin{array}{l}2 \text { Docentes } \\
1 \text { Assisten- } \\
\text { cial }\end{array}$ & Brasil & $\begin{array}{l}2 \text { Doutores } \\
1 \text { Mestre }\end{array}$ \\
\hline $\begin{array}{l}\text { Matsukura, } \\
\text { Tanõ, } 2014\end{array}$ & $\begin{array}{l}2 \text { Terapeutas } \\
\text { Ocupacionais }\end{array}$ & $\begin{array}{l}1 \text { Docente } \\
1 \text { Assisten- } \\
\text { cial }\end{array}$ & Brasil & $\begin{array}{l}1 \text { Doutor } \\
1 \text { Mestre }\end{array}$ \\
\hline $\begin{array}{l}\text { Noronha et } \\
\text { al., } 2016\end{array}$ & $\begin{array}{l}6 \text { Enfer- } \\
\text { meiros }\end{array}$ & $\begin{array}{l}6 \text { Assisten- } \\
\text { ciais }\end{array}$ & Brasil & 6 Graduados \\
\hline $\begin{array}{l}\text { Santos de } \\
\text { Aguiar Jr, } \\
\text { de Oliveira, } \\
\text { \& Almeida } \\
\text { de Araújo, } \\
2015\end{array}$ & $\begin{array}{l}1 \text { Enfer- } \\
\text { meiros } \\
1 \text { Farmacêu- } \\
\text { tico } \\
1 \text { Psicólogo }\end{array}$ & $\begin{array}{l}3 \text { Assisten- } \\
\text { ciais }\end{array}$ & Brasil & 3 Mestres \\
\hline $\begin{array}{l}\text { Stringer et } \\
\text { al., 2015a }\end{array}$ & $\begin{array}{l}2 \text { Enfer- } \\
\text { meiros } \\
2 \text { Psicólogos }\end{array}$ & 4 Docentes & Holanda & 4 Doutores \\
\hline $\begin{array}{l}\text { Stringer et } \\
\text { al., 2015b }\end{array}$ & $\begin{array}{l}3 \text { Enfer- } \\
\text { meiros } \\
3 \text { Psicólogos }\end{array}$ & 6 Docentes & Holanda & $\begin{array}{l}2 \text { Doutores } \\
2 \text { Mestres } \\
2 \text { Especial- } \\
\text { istas }\end{array}$ \\
\hline $\begin{array}{l}\text { Tszesnioski } \\
\text { et al., } 2015\end{array}$ & $\begin{array}{l}1 \text { Enfermeiro } \\
2 \text { Terapeutas } \\
\text { Ocupacionais } \\
1 \text { Fono- } \\
\text { audiólogo }\end{array}$ & $\begin{array}{l}4 \text { Assisten- } \\
\text { ciais }\end{array}$ & Brasil & $\begin{array}{l}2 \text { Especial- } \\
\text { istas } \\
2 \text { Graduados }\end{array}$ \\
\hline $\begin{array}{l}\text { Zegwaard } \\
\text { et al., } 2017\end{array}$ & $\begin{array}{l}4 \text { Enfer- } \\
\text { meiros }\end{array}$ & 4 Docentes & Holanda & 4 Doutores \\
\hline
\end{tabular}

Fonte: Dados da Pesquisa

\section{Caracterização das publicações}

Do total de publicações, $53 \%(n=8)$ encontram-se na BVS e, 47\% ( $n=7)$, na EBSCO. Maior ocorrência de publicações deu-se em 2015 (53\%, n=8). Mais da metade publicados na língua portuguesa $(53 \%, \mathrm{n}=8)$ e, em periódicos diversos, associados às profissões da área de saúde. Quanto ao delineamento, apenas um estudo com abordagem quantitativa e, 93,5\% $(n=14)$, são pesquisas qualitativas que envolvem estudos de caso, relatos de experiência, pesquisa ação, convergente assistencial e representações sociais (Quadro 2).
Quadro 2 - Caracterização das publicações analisadas

\begin{tabular}{|c|c|c|c|c|c|}
\hline Autor & Base & Ano & Periódico & Idioma & $\begin{array}{l}\text { Delinea- } \\
\text { mento }\end{array}$ \\
\hline $\begin{array}{l}\text { Bark- } \\
\text { mann et } \\
\text { al., } 2015\end{array}$ & EBSCO & 2015 & $\begin{array}{l}\text { European } \\
\text { Child \& } \\
\text { Adolescent } \\
\text { Psychiatry }\end{array}$ & Inglês & $\begin{array}{l}\text { Coorte } \\
\text { prospec- } \\
\text { tiva }\end{array}$ \\
\hline $\begin{array}{l}\text { Beentjes } \\
\text { et al., } \\
2016\end{array}$ & EBSCO & 2016 & $\begin{array}{l}\text { Perspectives } \\
\text { in Psychiat- } \\
\text { ric Care }\end{array}$ & Inglês & $\begin{array}{l}\text { Descritivo, } \\
\text { qualitativo }\end{array}$ \\
\hline $\begin{array}{l}\text { Dutra et } \\
\text { al., } 2017\end{array}$ & BVS & 2017 & $\begin{array}{l}\text { Escola Anna } \\
\text { Nery }\end{array}$ & $\begin{array}{l}\text { Portu- } \\
\text { guês }\end{array}$ & $\begin{array}{l}\text { Pesquisa } \\
\text { Conver- } \\
\text { gente As- } \\
\text { sistencial }\end{array}$ \\
\hline $\begin{array}{l}\text { Gloff et } \\
\text { al., } 2015\end{array}$ & EBSCO & 2015 & $\begin{array}{l}\text { International } \\
\text { Review of } \\
\text { Psychiatry }\end{array}$ & Inglês & $\begin{array}{l}\text { Descritivo, } \\
\text { qualitativo }\end{array}$ \\
\hline $\begin{array}{l}\text { Hirota et } \\
\text { al., } 2015\end{array}$ & EBSCO & 2015 & $\begin{array}{l}\text { Psychiatry } \\
\text { and Clinical } \\
\text { Neurosci- } \\
\text { ences }\end{array}$ & Inglês & $\begin{array}{l}\text { Descri- } \\
\text { tivo, quan- } \\
\text { titativo }\end{array}$ \\
\hline $\begin{array}{l}\text { Macedo } \\
\text { Monteiro } \\
\text { et al., } \\
2015 \\
\end{array}$ & BVS & 2015 & $\begin{array}{l}\text { Pesquisa } \\
\text { Cuidado é } \\
\text { Fundamen- } \\
\text { tal Online }\end{array}$ & $\begin{array}{l}\text { Portu- } \\
\text { guês }\end{array}$ & $\begin{array}{l}\text { Descritivo, } \\
\text { qualitativo }\end{array}$ \\
\hline $\begin{array}{l}\text { Ma- } \\
\text { chineski } \\
\text { et al., } \\
2013\end{array}$ & BVS & 2013 & $\begin{array}{l}\text { Revista } \\
\text { Gaúcha de } \\
\text { Enfermagem }\end{array}$ & $\begin{array}{l}\text { Portu- } \\
\text { guês }\end{array}$ & $\begin{array}{l}\text { Descritivo, } \\
\text { qualitativo }\end{array}$ \\
\hline $\begin{array}{l}\text { Martinez } \\
\text { et al., } \\
2013\end{array}$ & BVS & 2013 & $\begin{array}{l}\text { Revista } \\
\text { Gaúcha de } \\
\text { Enfermagem }\end{array}$ & $\begin{array}{l}\text { Portu- } \\
\text { guês }\end{array}$ & $\begin{array}{l}\text { Repre- } \\
\text { sentações } \\
\text { sociais } \\
\end{array}$ \\
\hline $\begin{array}{l}\text { Matsu- } \\
\text { kura, } \\
\text { Tanõ, } \\
2014\end{array}$ & BVS & 2014 & $\begin{array}{l}\text { Revista de } \\
\text { Terapia } \\
\text { Ocupacional } \\
\text { da Universi- } \\
\text { dade de São } \\
\text { Paulo } \\
\end{array}$ & $\begin{array}{l}\text { Portu- } \\
\text { guês }\end{array}$ & $\begin{array}{l}\text { Descritivo, } \\
\text { qualitativo }\end{array}$ \\
\hline $\begin{array}{l}\text { Noronha } \\
\text { et. al., } \\
2016 \\
\end{array}$ & BVS & 2016 & $\begin{array}{l}\text { Revista } \\
\text { Gaúcha de } \\
\text { Enfermagem }\end{array}$ & $\begin{array}{l}\text { Portu- } \\
\text { guês }\end{array}$ & $\begin{array}{l}\text { Descritivo, } \\
\text { qualitativo }\end{array}$ \\
\hline $\begin{array}{l}\text { Santos de } \\
\text { Aguiar } \\
\text { Jr et al., } \\
2015 \\
\end{array}$ & BVS & 2015 & $\begin{array}{l}\text { Pesquisa } \\
\text { Cuidado é } \\
\text { Fundamen- } \\
\text { tal Online } \\
\end{array}$ & $\begin{array}{l}\text { Portu- } \\
\text { guês }\end{array}$ & $\begin{array}{l}\text { Relato de } \\
\text { experiên- } \\
\text { cia }\end{array}$ \\
\hline $\begin{array}{l}\text { Stringer } \\
\text { et al., } \\
2015 a\end{array}$ & EBSCO & $2015 a$ & $\begin{array}{l}\text { Perspectives } \\
\text { in Psychiat- } \\
\text { ric Care }\end{array}$ & Inglês & $\begin{array}{l}\text { Estudo de } \\
\text { caso }\end{array}$ \\
\hline $\begin{array}{l}\text { Stringer } \\
\text { et al., } \\
2015 b\end{array}$ & EBSCO & $2015 b$ & $\begin{array}{l}\text { Perspectives } \\
\text { in Psychiat- } \\
\text { ric Care }\end{array}$ & Inglês & $\begin{array}{l}\text { Estudo de } \\
\text { caso }\end{array}$ \\
\hline $\begin{array}{l}\text { Tszesn- } \\
\text { ioski et } \\
\text { al., } 2015\end{array}$ & BVS & 2015 & $\begin{array}{l}\text { Ciência \& } \\
\text { Saúde Cole- } \\
\text { tiva }\end{array}$ & $\begin{array}{l}\text { Portu- } \\
\text { guês }\end{array}$ & $\begin{array}{l}\text { Pesquisa- } \\
\text { ação }\end{array}$ \\
\hline $\begin{array}{l}\text { Zeg- } \\
\text { waard et } \\
\text { al., } 2017\end{array}$ & EBSCO & 2017 & $\begin{array}{l}\text { BMC Psy- } \\
\text { chiatry }\end{array}$ & Inglês & $\begin{array}{l}\text { Descritivo, } \\
\text { qualitativo }\end{array}$ \\
\hline
\end{tabular}


Atuação do enfermeiro diante de crianças e adolescentes em sofrimento mental

As categorias temáticas foram assim nomeadas: a organização da assistência ao público infantojuvenil em sofrimento mental, o olhar ampliado nas relações com crianças e adolescentes em sofrimento mental, a promoção da autonomia e ressocialização diante do sofrimento mental e, a inclusão do cuidador/familiar no planejamento dos cuidados.

\section{A organização da assistência ao público infantojuve- nil em sofrimento mental}

Na gestão do cuidado da criança e do adolescente em sofrimento mental a sistematização da assistência de enfermagem (SAE) revela-se ferramenta eficaz, ao oferecer fundamentos teóricos para o alcance dos objetivos (Macedo Monteiro et al., 2015). Outras atividades são gerir os cuidados colaborativos, planear as ações intersetoriais e elaborar os projetos terapêuticos singulares (Matsukura, Taño, 2014; Stringer et al., 2015a).

Pontualmente, o plano terapêutico é metodologia assistencial que cria alvos reais e atingíveis, além de auxiliar os cuidadores na percepção de alterações comportamentais, o que torna eficiente a procura pelos serviços de saúde (Gloff et al., 2015; Macedo Monteiro et al., 2015). O planeamento descreve comportamentos específicos em cada estágio patológico e apresenta possíveis soluções para o seu controle. O contato precoce com o serviço de saúde, nas recaídas, torna o feedback mais objetivo (Beentjes et al., 2016). O cotidiano do enfermeiro envolve, pois, ações sistematizadas em equipa, baseadas em competências para conhecer o indivíduo (Gloff et al., 2015; Macedo Monteiro et al., 2015). De fato, o conhecimento e habilidades são fundamentais para maior precisão, planeamento adequado e desenvolvimento de ações apropriadas e consistentes de controle e manutenção que envolvam não apenas o indivíduo mas, também, a sua família (Barkmann et al., 2015; Naughton et al., 2018).

A atuação do enfermeiro no CAPSi considera a redução dos custos e a maximização da eficiência do cuidado, a partir do levantamento dos recursos e pontos fortes. O julgamento clínico permite prever os diagnósticos de enfermagem e planear cuidados que promovam a saúde e previnam problemas (Macedo Monteiro et al., 2015). Dominar as ferramentas disponíveis descortina a dimensão mais abrangente e relacional no cuidado à saúde mental dos jovens.
O olhar ampliado nas relações com crianças e adolescentes em sofrimento mental

As abordagens diferenciadas individualizam o cuidado, quando o modelo assistencial, até então denominado hospitalocêntrico, deu lugar a investimentos na dimensão social e histórica do indivíduo, pois as relações ultrapassaram os serviços de saúde (Dutra et al., 2017). Porém, no cenário atual, ainda predomina a heterogeneidade cultural, religiosa e econômica, que desmotiva impor modelos com foco nas relações terapêuticas (Beentjes et al., 2016), adaptadas à cultura do local e da criança (Hirota et al., 2015), a partir do acolhimento/ acompanhamento que favorecem a inserção social (Matsukura, Taño, 2014).

Não se discute que o acolhimento é uma das principais atitudes para qualificar e humanizar o atendimento (Santos de Aguiar Jr et al., 2015). O enfermeiro valoriza o ser social, quando conhece a sua história e a articula com a sua família e ambiente vivido, suas redes de apoio e desejos (Beentjes et al., 2016; Dutra et al., 2017; Macedo Monteiro et al., 2015).

Não obstante, os enfermeiros ainda transitam nos discursos sobre a loucura e convivem, ao mesmo tempo, com o objetivo e o subjetivo, razão e paixão. Competências que favorecem o olhar crítico em saúde mental (Macedo Monteiro et al., 2015; Stringer et al., 2015a).

Não se pode deixar de destacar que, no âmbito das relações, a comunicação é a estratégia para o olhar diferenciado e para o estabelecimento de vínculos e revela-se pelo toque e pelo contato físico, ao relacionar afeto e segurança. As oficinas terapêuticas permitem ao outro se manifestar para que se amplie o processo de construção do viver com dignidade e da cidadania, mesmo diante de sofrimento mental. E o lúdico, em especial, marca presença, no sentido de fazer emergir o simbólico e revelar pensamentos relacionais (Dutra et al., 2017; Gloff et al., 2015; Macedo Monteiro et al., 2015; Martinez et al., 2013). Portanto, a utilização de ferramentas específicas concorrem para o cuidado autêntico e para a autonomia.

\section{A promoção da autonomia e ressocialização diante do sofrimento mental}

A corresponsabilidade no cuidado e a reinserção social dos jovens na família e na sociedade, requerem atuação multiprofissional, proporcionam bem estar biopsicossocial e promovem cidadania (Macedo Monteiro et al., 2015). 
E os enfermeiros apostam que mediar relacionamentos livres de julgamento contribui para a autonomia, o fortalecimento da vida social e a construção de identidades. Estabelecem-se afinidades entre a autonomia e a educação para a saúde por meio de intervenções planejadas (Beentjes et al., 2016; Dutra et al., 2017).

Entretanto, o CAPSi demanda uma estratégia de intervenção específica, que considera as características subjetivas, físicas e jurídicas diferenciadas, integrada à rede de saúde, educação, assistência social e garantia de direitos. O cuidado deve ultrapassar o tratamento dos transtornos mentais (modelo centrado na doença) e buscar a inclusão social, a integralidade e a qualidade de vida (Santos de Aguiar Jr et al., 2015; Tszesnioski et al., 2015). A saúde mental dos jovens constitui-se relação social complexa que precisa de ser pensada, planeada e mediada, para promover o prota $\neg$ gonismo dos indivíduos, autonomia e consciência crítica da realidade de vida (Dutra et al., 2017; Noronha et al., 2016).

Nessa perspectiva, o cuidado autônomo significa conviver, experimentar, provocar situações desejadas, potencialmente veladas nas histórias de sofrimento mental (Dutra et al., 2017). Significa ponte para exercitar o viver, pois os limites e as possibilidades são reconhecidos, estabelecem-se laços de cuidado consigo, com o trabalho e com a afetividade no entorno. Para o enfermeiro o sofrimento mental não atrapalha reconhecer potencialidades individuais no cotidiano vivido (Dutra et al., 2017; Santos de Aguiar Jr et al., 2015).

Não menos importante, o envolvimento familiar para o autocuidado, é essencial, principalmente, diante de eventuais limitações das crianças e adolescentes, oportunidade em que os enfermeiros apostam na autonomia e na liberdade como fundamentos do cuidado (Macedo Monteiro et al., 2015). A colaboração eficaz e precoce entre indivíduo, profissionais e cuidadores informais é um dos objetivos na assistência, mas o próprio jovem, é responsável pelos seus caminhos. Fato que evidencia e reforça a promoção da autonomia e tomada de decisão (Beentjes et al., 2016; Stringer et al., 2015b). Assim, o cuidado autêntico não se furta de considerar os familiares na idealização de intervenções em saúde mental.

\section{A inclusão do cuidador/familiar no planeamento dos cuidados}

Crianças e adolescentes percebem, na família, o apoio necessário, elemento chave para auxiliar, precocemente, a equipe de saúde sobre a realidade para a definição do plano terapêutico que se antecipe a quadros agudos (Beentjes et al., 2016; Martinez et al., 2013).
O grupo familiar apresenta-se como peça fundamental na adesão e resposta às terapias propostas, por se envolver no cuidado (Noronha et al., 2016; Santos de Aguiar Jr et al., 2015).

A intervenção junto dos cuidadores ocorre de forma paralela ao tratamento do jovem, quando a complexidade das relações demanda aconselhamento e apoio fundamentados (Hirota et al., 2015; Naughton et al., 2018; Zegwaard et al., 2017), pois nessa fase, torna-se possível a compreensão da situação vivenciada. E, ao profissional, cabe vislumbrar o ambiente relacional (Machineski et al., 2013). A escuta cuidadosa, somada ao apoio sem julgamento, conforta o familiar, ocasião em que a empatia é a ferramenta de aproximação. Quando há informação e compreensão a carga tende a diminuir e o resultado da inteiração, cuja precocidade se deseja, representa a continuidade e a coordenação de atendimento melhorado e cada vez mais pontual (Beentjes et al., 2016; Dutra et al., 2017; Stringer et al., 2015a; Zegwaard et al., 2017).

Entre os métodos desenvolvidos para a aceitação e o enfrentamento da situação, destacam-se a educação em saúde, treinamentos, grupos de apoio e aconselhamento (Machineski et al., 2013; Zegwaard et al., 2017). Os familiares acreditam que o enfermeiro da saúde mental busca o melhor para o indivíduo e para o cuidador, o que reforça a ideia de deferimento, fragilidade respeitada, confiança não violada e promessas mantidas (Zegwaard et al., 2017).

Emerge, portanto, a necessidade de ações coordenadas para a interação e a comunicação entre o enfermeiro, o jovem e sua família, consideradas relevantes para a adesão ao tratamento e a reinserção social efetiva (Martinez et al., 2013; Naughton et al., 2018).

Isso posto, os resultados apontaram que a atuação do enfermeiro perpassa pela gestão e organização do cuidado individualizado, com vistas à promoção da autonomia e ressocialização desses jovens e suas famílias.

\section{DISCUSSÃO}

Os achados desta revisão vão de encontro a outros estudos que abordam o quanto a enfermagem colabora no tratamento da criança e do adolescente em sofrimento mental. Destacam-se a utilização de metodologias como a SAE e o olhar mais abrangente que alcance familiares e cuidadores. O processo de trabalho do enfermeiro compõe-se de duas esferas distintas e, ao mesmo tempo, complementares: assistir/cuidar e administrar/gerir a atenção ao jovem em sofrimento mental. Na esfera assistencial, considera-se o cuidado integral. 
Do ponto de vista gerencial, a sua atuação almeja a organização dos processos de trabalho e a adequação dos recursos humanos em enfermagem, ao criar e implementar condições apropriadas para o desempenho de sua equipe nas ações assistenciais (Senna et al., 2014). Para atingir tais dimensões o profissional precisa de se preparar, pois a administração e a gestão da assistência relacionam-se à SAE, ferramenta que programa e avalia os cuidados com vistas à redução de possíveis danos e à recuperação do indivíduo na atenção psiquiátrica (Peixoto et al., 2017). Torna-se possível afirmar que mais que as habilidades técnicas, os resultados demonstram a necessidade de ampliação do olhar profissional para além do corpo físico das crianças e adolescentes em sofrimento mental. Há que se considerar o contexto, os atores envolvidos e as relações aí constituídas no estabelecimento dos planos de cuidado. Os estudos selecionados asseveraram, de maneira categórica, que a garantia da qualidade assistencial ao público específico, depende da competência do enfermeiro para a condução individualizada da atenção em saúde mental. Estariam, portanto, envolvidas as habilidades baseadas no conhecimento técnico-científico e, não menos importante, no campo do subjetivo.

Ao delinear os desafios dos saberes e práticas de enfermeiros na saúde mental, diante da reforma psiquiátrica, pesquisadores afirmam a complexidade da assistência, pela especificidade de cada jovem que sofre mentalmente. Defendem o olhar voltado para a qualidade do tratamento e para o respeito à dignidade do indivíduo em sofrimento (Souza, Afonso, 2015). Igualmente, o cuidado com bases em diagnósticos psiquiátricos de enfermagem, ultrapassa procedimentos associados à administração de medicamentos, higiene, alimentação e controle físico ou contenção. Incluem-se atividades terapêuticas multidisciplinares e reinserção do indivíduo em seu contexto, para maior qualidade de vida (Peixoto et al., 2017).

O diferencial na atuação do enfermeiro reside nas mudanças realizadas a partir do saber e do fazer. O profissional torna-se um líder na implantação das novas políticas de saúde mental. Ao sistematizar e organizar o serviço reveste-se de mediador entre o indivíduo, a equipe, a família e a comunidade, circunstância que concorre para a resolutividade e o enfrentamento mais efetivos de problemas e agravos à saúde (Bortone, Wingester, 2016; Naughton et al., 2018).
De fato, um estudo sobre as estratégias de cuidado no CAPSi, a partir de concepções de familiares e profissionais, faz emergir que as práticas valorizam, sobretudo, a formação de vínculo e a subjetividade do indivíduo. Passa a ser considerado um ser expressivo, criativo, lúdico, social, com capacidade de desenvolvimento funcional, de forma autônoma e de acordo com suas possibilidades (Henriques Araújo et al., 2015). Significa promover a saúde mental infantojuvenil na perspectiva da psiquiatria social (Gogineni, Rothe, \& Pumariega, 2019).

Pelos resultados a ressocialização integra o papel do enfermeiro, ao favorecer e estimular a autonomia, pois a assistência ultrapassa o silenciamento de sintomas e o isolamento do sofrimento mental. Há que haver a superação de estigmas, de preconceitos e de estereótipos vinculados à loucura. Vislumbra-se o olhar marcado pela defesa dos direitos humanos e pelo resgate da cidadania (Zeferino et al., 2016).

Essa revisão evidencia tantos outros estudos ao enaltecer o desempenho da família no contexto assistencial. O cuidado que liberta, tão relevante na ressocialização, inclui o entorno do indivíduo marcado pelo sofrimento mental e considera as informações decorrentes do vínculo estabelecido. São elementos que dão suporte e embasam as ações assistenciais (Cunha et al., 2017).

A atuação do enfermeiro frente a crianças e adolescentes que sofrem mentalmente é complexa e essencial para a qualidade de vida dos jovens. A complexidade deve-se à variedade de intervenções e de olhares advindos do conhecimento técnico e científico em psiquiátrica infantojuvenil, à gestão do cuidado individual, toque e comunicação, até a promoção da autonomia e da cidadania, sem deixar de incluir e valorizar os familiares. Entretanto, a presente revisão não encerra questões sobre o cuidado em saúde mental, especificamente, de crianças e adolescentes. O público carece de respostas a outras questões que emergirão dos resultados. A visão dos profissionais desse cuidado específico poderá fazer despontar outras dimensões. E, a participação dos familiares pode evidenciar as dificuldades cotidianas na busca pela saúde mental de seus integrantes mais jovens. 


\section{CONCLUSÕES}

O enfermeiro é o profissional que detém as competências exigidas para mesclar assistência e gestão em quaisquer níveis de cuidado em saúde, porém torna-se essencial e urgente o suprimento das lacunas ainda existentes para alcançar resultados satisfatórios na atenção a crianças e adolescentes em sofrimento mental. Não se pode deixar de ressaltar o papel das instituições de ensino na promoção do conhecimento aberto, reflexivo, curioso para se buscar, além do aprender, o reaprender a aprender. Conhecimentos, habilidades e atitudes para planear as ações em saúde para público tão peculiar demandam considerar dimensões relacionais e contextuais, em especial as que envolvem a família. Analisar o papel do enfermeiro fez emergir o profissional articulador do cuidado ao público infantojuvenil em sofrimento mental, ao assistir, gerenciar e promover o cuidado autônomo e integral, com vistas à ressocialização. Prática cotidiana complexa e fundamental que liberta e associa-se a ações e intervenções capazes de incluir o jovem e seu entorno no plano terapêutico. Entretanto, novos estudos devem contemplar a visão da família, da escola e da vizinhança daquele que sofre mentalmente.

\section{IMPLICAÇÕES PARA A PRÁTICA CLÍNICA}

O permanente aprimoramento do enfermeiro para alcançar padrões assistenciais de excelência de crianças e adolescentes em sofrimento mental concorre para a de vínculos mais fortes e consistentes, além de planos terapêuticos mais efetivos. Repercussão não menos importante estabelece-se no apoio aos familiares dos jovens, ponto chave, por permitir a inclusão e corresponsabilização na manutenção da saúde e no enfrentamento dos conflitos relacionais. Esse contorno permitiria assistir crianças e adolescentes em sofrimento mental no processo de aceitação, autonomia e reinserção familiar e social.

\section{REFERÊNCIAS BIBLIOGRÁFICAS}

Barkmann, C., Otto, C., Schön, G., Schulte-Markwort, M., Schlack, R., Ravens-Sieberer, U., Klasen F., \& The BELLA study group. (2015). Modelling trajectories of psychosomatic health complaints in children and adolescents: results of the BELLA study. European Child \& Adolescent Psychiatry, 24(6), 685-694. Disponível em: https://link.springer.com/article/10.1007/s00787-0140656-2
Beentjes, T. A., Goossens, P. J., \& Jongerden, I. P. (2016). Nurses' experience of maintaining their therapeutic relationship with outpatients with bipolar disorder and their caregivers during different stages of a manic episode: a qualitative study. Perspectives in Psychiatric Care, 52(2), 131-138. Doi: 10.1111/ppc.12109

Bortone, A. R. T., \& Wingester, E. L. C. (2016). Identificação do espectro do transtorno autista durante o crescimento e o desenvolvimento infantil: o papel do profissional de enfermagem. SynThesis Revista Digital FAPAM, 7(7), 131-148. Disponível em https://periodicos.fapam.edu.br/index.php/synthesis/article/view/133

Botelho, L. L. R., Cunha, C. C. A., \& Macedo, M. (2011). O método da revisão integrativa nos estudos organizacionais. Gestão e sociedade, 5(11), 121-136. Doi: 10.21171/ges.v5i11.1220

Cunha, M. P., Borges, L. M., \& Bezerra, C. B. (2017). Infância e saúde mental: perfil das crianças usuárias do Centro de Atenção Psicossocial Infantil. MudançasPsicologia da Saúde, 25(1), 27-35. Doi: 10.15603/21761019/mud.v25n1p27-35

Delfini, P. S. S., \& Reis, A. O. A. (2012). Articulação entre serviços públicos de saúde nos cuidados voltados à saúde mental infantojuvenil. Cadernos de Saúde Pública, 28(2), 357-366. Doi: 10.1590/S0102311X2012000200014

Dutra, V. F. D., Bossato, H. R., \& Oliveira, R. M. P. (2017). Mediar a autonomia: um cuidado essencial em saúde mental. Escola Anna Nery, 21(3): e20160284. Doi: 10.1590/2177-9465-ean-2016-0284

Gloff, N. E., LeNoue, S. R., Novins, D. K., \& Myers, K. (2015). Telemental health for children and adolescents. International Review of Psychiatry, 27(6), 513-524. Doi: $10.3109 / 09540261.2015 .1086322$

Gogineni, R. R., Rothe, E. M., \& Pumariega, A. J. (2019). Setting the global agenda for social psychiatry: child and adolescent psychiatric perspectives. World Social Psychiatry, 1(1), 53-61. Doi: 10.4103/WSP.WSP_12_19 
Henriques Araújo, G., Mendonça Saraiva, A., Pinheiro de Carvalho, M., Castro Gomes, A., de Faria Pimentel Costa, L., \& de Oliveira Ferreira Filha, M. (2015). Estratégias de cuidado desenvolvidas no CAPS infantil: concepções de familiares e profissionais. Revista de Pesquisa Cuidado é Fundamental Online, 7(Supl), 28-38. Disponível em https://www.redalyc.org/pdf/5057/505750949003. pdf

Hirota, T., Guerrero, A., Sartorius, N., Fung, D., Leventhal, B., Ong, S. H., Kaneko, H., Kim, B., Cho, S. C., Skokauskas, N., \& Consortium on Academic Child and Adolescent Psychiatry in the Far East (CACAP FE). (2015). Child and adolescent psychiatry in the Far East. Psychiatry and Clinical Neurosciences, 69(3), 171-177. Doi: $10.1111 /$ pcn. 12800

Macedo Monteiro, A. R., Queiróz Martins, M. G., Aguiar Lobô, S., Araújo de Freitas, P. C., Morais Barros, K., \& do Vale Tavares Fátima Tavares, S. F. (2015). Sistematização da assistência de enfermagem à criança e ao adolescente em sofrimento psíquico. Revista de Pesquisa Cuidado é Fundamental Online, 7(4), 3185-3196. Disponível em: https://www.redalyc.org/pdf/5057/505750948004.pdf

Machado, C. M., Luiz, A. M. A. G., Marques Filho, A. B., Miyazaki, M. C. O. S., Domingos, N. A. M., \& Cabrera, E. M.S. (2014). Ambulatório de psiquiatria infantil: prevalência de transtornos mentais na infância. Revista Psicologia: Teoria e Prática, 16(2), 53-62. Disponível em: http://pepsic.bvsalud.org/pdf/ptp/v16n2/05.pdf

Machineski, G. G., Schneider, J. F., \& Camatta, M. W. (2013). O tipo vivido de familiares de usuários de um centro de atenção psicossocial infantil. Revista Gaúcha de Enfermagem, 34(1), 126-132. Doi: 10.1590/S198314472013000100016

Martinez, E. A., Tocantins, F. R., \& Souza, S. R. (2013). As especificidades da comunicação na assistência de enfermagem à criança. Revista Gaúcha de Enfermagem, 34(1), 37-44. Doi: 10.1590/S1983-14472013000100005

Matsukura, T. S., \& Tanõ, B. L. (2014). Os centros de atenção psicossocial infantojuvenis: características organizacionais e oferta de cuidados. Revista de Terapia Ocupacional da Universidade de São Paulo, 25(3), 208216. Doi: 10.11606/issn.2238-6149.v25i3p208-216
Mendes, K. D. S., Silveira, R. C. C. P., \& Galvão, C. M. (2008). Revisão integrativa: método de pesquisa para a incorporação de evidências na saúde e na enfermagem. Texto \& Contexto - Enfermagem, 17(4), 758-764. Doi: 10.1590/S0104-07072008000400018

Ministério da Saúde. (2002). Portaria n. 336, de 19 de fevereiro de 2002. Define e estabelece diretrizes para o funcionamento dos Centros de Atenção Psicossocial do Ministério da Saúde. Diário Oficial da União. Brasília, DF: Ministério da Saúde. Disponível em: http://bvsms.saude.gov.br/bvs/saudelegis/gm/2002/ prt0336_19_02_2002.html

Naughton, M. F. A., Maybery, D. J., Goodyear, M. (2018). Prevalence of mental illness within families in a regional child-focussed mental health service. Internacional Journal of Mental Health Nursing, 27(2), 901-910. Doi: 10.1111/inm. 12386

Noronha, A. A., Folle, D., Guimarães, A. N., Brum, M. L. B., Schneider, J.F., \& Motta, M. G. C. (2016). Percepções de familiares de adolescentes sobre oficinas terapêuticas em um centro de atenção psicossocial infantil. Revista Gaúcha de Enfermagem, 37(4), e56061. Doi: 10.1590/1983-1447.2016.04.56061

Peixoto, M. S. S., Gonzaga, M. F., Marques, C. S. F., Silva, J. O. M., \& Souza-Neto, C. M. (2017, maio). Os Cuidados de Enfermagem com Base no Diagnóstico de Enfermagem Psiquiátrico. Anais do $2^{\circ}$ Congresso Internacional de Enfermagem, Fortaleza, CE, Brasil. Disponível em: https://eventos.set.edu.br/index.php/cie/article/ view/5548

Reis, A. O. A., Delfini, P. S. S., Dombi-Barbosa, C., \& Bertolino Neto, M. M. (2010). Breve história da saúde mental infantojuvenil. In Atenção em saúde mental para crianças e adolescentes no SUS. São Paulo: Hucitec.

Santos de Aguiar Jr, V., de Oliveira, A., \& Almeida de Araújo, L. (2015). Hygiene and mental health: body care in the clinical intervention in a CAPSI. Revista de Pesquisa Cuidado é Fundamental Online, 7(2), 25822590. Disponível em: http://seer.unirio.br/index.php/ cuidadofundamental/article/view/3620 
Secretaria de Atenção à Saúde. (2005a). Reforma psiquiátrica e política de saúde mental no Brasil. Apresentação na Conferência Regional de Reforma dos Serviços de Saúde Mental: 15 anos depois de Caracas. Coordenação Geral de Saúde Mental. Ministério da Saúde. Brasília, DF. Disponível em: http://bvsms.saude.gov.br/ bvs/publicacoes/Relatorio15_anos_Caracas.pdf

Secretaria de Atenção à Saúde. (2005b). Caminhos para uma política de saúde mental infantojuvenil. Departamento de Ações Programáticas Estratégicas. Ministério da Saúde. Brasília, DF. Disponível em: http://portalarquivos2.saude.gov.br/images/pdf/2015/marco/10/ Caminhos-para-uma-Politica-de-Sa--de-Mental-Infanto-Juvenil--2005-.pdf

Secretaria de Atenção à Saúde. (2015). Saúde Mental em Dados $n^{\circ}$ 12. Boletim Informativo. Departamento de Ações Programáticas Estratégicas. Ministério da Saúde. Brasília, DF. Disponível em: http://portalarquivos. saude.gov.br/images/pdf/2015/dezembro/15/Preven---o-em-Dados--2013-2015-.pdf

Secretaria Executiva. (2003). A Política do Ministério da Saúde para atenção integral a usuários de álcool e outras drogas. Coordenação Nacional de DST/Aids. Ministério da Saúde. Brasília, DF. Disponível em: http:// bvsms.saude.gov.br/bvs/publicacoes/politica_atencao_ alcool_drogas.pdf

Senna, M. H., Drago, L. C., Kirchner, A. R., Santos, J. L. G., Erdmann, A. L., \& Andrade, S. R. (2014). Significados da gerência do cuidado construídos ao longo da formação profissional do enfermeiro. Revista da Rede de Enfermagem do Nordeste, 15(2),196-205. Doi: 10.15253/2175-6783.2014000200003

Souza, M. C., \& Afonso, M. L. M. (2015). Saberes e práticas de enfermeiros na saúde mental: desafios diante da Reforma Psiquiátrica. Gerais, Revista Interinstitucional de Psicologia, 8(2), 332-347. Disponível em: http://pepsic.bvsalud.org/pdf/gerais/v8n2/v8n2a04.pdf
Stringer, B., van Meijel, B., Karman, P., Koekkoek, B., Hoogendoorn, A. W., Kerkhof, A. J., \& Beekman A. T. (2015a). Collaborative care for patients with severe personality disorders: preliminary results and active Ingredients from a pilot study (Part I). Perspectives in Psychiatric Care, 51(3), 189-189. Doi: 10.1111/ppc.12079

Stringer, B., van Meijel, B., Karman, P., Koekkoek, B., Kerkhof, A. J., \& Beekman, M. D. (2015b). Collaborative care for patients with severe personality disorders: analyzing the execution process in a pilot study (Part II). Perspectives in Psychiatric Care, 51(3), 220-227. Doi: 10.1111/ppc. 12087

The Joanna Briggs Institute. (2014). Joanna Briggs Institute reviewers' Manual: 2014 edition Adelaide, Austrália. Disponível em: http://joannabriggs.org/assets/ docs/sumari/reviewersmanual-2014.pdf

Thiengo, D. L., Cavalcante, M. T., \& Lovisi, G. M. (2014). Prevalência de transtornos mentais entre crianças e adolescentes e fatores associados: uma revisão sistemática. Jornal Brasileiro de Psiquiatria, 63(4), 360-372. Doi: 10.1590/0047-2085000000046

Tszesnioski, L. C., Nóbrega, K. B. G., Lima, M. L. L. T., \& Facundes, V. L. D. (2015). Construindo a rede de cuidados em saúde mental infantojuvenil: intervenções no território. Ciência \& Saúde Coletiva, 20(2), 363-370. Doi: 10.1590/1413-81232015202.05082014

Zeferino, M. T., Cartana, M. H. F., Fialho, M. B., Huber, M. Z., \& Bertoncello, K. C. G. (2016). Percepção dos trabalhadores da saúde sobre o cuidado às crises na rede de atenção psicossocial. Escola Anna Nery, 20(3): e20160059. Doi: 10.5935/1414-8145.20160059

Zegwaard, M. I., Aartsen, M. J., Grypdonck, M. H., \& Cuijpers, P. (2017). Trust: an essential condition in the application of a caregiver support intervention in nursing practice. BMC Psychiatry, 17(1), 47. Doi: 10.1186/ s12888-017-1209-2 